[Aus dem staatlichen hygienischen Institut zu Hamburg.]

\title{
Ueber Kochapparate für bedingt gesundheitsschädliches Fleisch und Versuche mit dem Hartmann'schen Fleischsterilisator.
}

Von

Dr. Rudolf Abel.

I.

Aufgabe der Flleischbeschau ist es, Fleisch von Schlachtthieren, dessen Genuss der menschlichen Gesundheit Schaden bringen kōnnte, vom Consum auszuschliessen. Von volkswirthschaftlichen Gesichtspunkten aus muss das Bestreben dabei aber dahin gerichtet sein, gesundheitsschädliches Fleisch von Schlachtvieh, soweit es durch besondere Arten der Behandlung sich seiner Gesundheitsgefährlichkeit entkleiden lässt, dem menschlichen Consum zu erhalten. So würde es vom öconomischen Standpunkte aus nicht gerechtfertigt sein, das Fleisch aller finnigen, trichinösen und schwerer erkrankten tuberculösen Thiere zum Beispiel, das sich in relativ einfacher Weise in ein ungefährliches Nahrungsmittel verwandeln lässt, unweigerlich dem Consum zu entziehen.

Derartigen Erwägungen Rechnung tragend, hat man sich bemüht, Mittel und Wege ausfindig zu machen, wie die Ausnutang solchen bedingt gesundheitsschädlichen Fleisches für den menschlichen Genuss ohne Gefahr für Leib und Leben des Consumenten möglich zu machen und zweckmässig zu gestalten ist.

Im Wesentlichen sind es drei Methoden, welche man anwenden kann, um das Fleisch kranker Thiere in ein ungefährliches Nahrungsmittel zu verwandeln, nämlich Pökeln, Räuchern und Kochen desselben. Während Pökeln und Räuchern bei rationeller Ausführung zur Abtödtung 
thierischer Parasiten im Fleisch, wie der Finnen und Trichinen, mit Sicherheit genügt, bietet es für die Vernichtung pathogener Bakterien im Fleisch keine hinreichende Gewähr. Fine gut ausgeführte Kochung aber liefert dieselbe. Daher sind Kochverfahren zur Befreiung des Fleisches von gesundheitsschädlichen Parasiten bakterieller Natur ganz besonders in Anwendung gezogen worden.

Der Gedanke, gesundheitsschädliches Fleisch, dessen Gefährlichkeit durch Kochen vernichtet werden kann, den Consumenten in rohem $\mathrm{Zu}$ stande auf der Freibank zu verabfolgen, aber mit dem Hinweis, dass es nur nach guter Durchkochung ohne Schaden genossen werden darf, muss von vornherein von der Hand gewiesen werden. Abgesehen von der Schwierigkeit, jedem Käufer die Wichtigkeit guter Durchkochung des Fleisches genügend deutlich zu machen, steht es bei der Gleichgültigkeit und Gedankenträgheit des Publikums in derartigen Dingen keineswegs fest, dass der gewarnte Käufer auch wirklich für ausreichende Kochung Sorge trägt und nicht etwa das Fleisch in mangelhaft gekochtem Zustande geniesst oder Andere geniessen lässt. Der Freibank sollte in rohem Zustande nur solches Fleisch zugehen, das aus irgend einem Grunde minderwerthig ist, der menschlichen Gesundheit aber auch bei Consumption in rohem oder mangelhaft gekochten Zustande nicht schädlich werden kann.

Aller Orten sieht man daher den Grundsatz sich Bahn brechen, die Kochung in rohem Zustande gesundheitsschädlichen Fleisches auf den Schlachthöfen selbst in geeigneten Apparaten nach zweckentsprechenden Verfahren und unter sachverständiger Controle vornehmen zu lassen und das Fleisch nur in gekochtem Zustande zu billigem Preise, der seine Minderwerthigkeit gegenüber bankwürdigem Fleische darthut, zum Verkaufe zuzulassen. Man trifft zweckmässig dabei noch die Einschränkung, das Fleisch nur in kleinen Portionen, von 2000 bis 4000 grm etwa, abzugeben, um einem Weiterverkauf desselben, seiner Verwendung in Speisewirthschaften u. s. w., nach Möglichkeit vorzubeugen.

Die zur Kochung bedingt gesundheitsschädlichen Fleisehes verwendbaren Kochapparate lassen sich in zwei Gruppen eintheilen, je nachdem in ihnen das Fleisch in Wasser oder im Dampf abgekocht wird.

Von den Apparaten, in welchen die Kochung des Fleisches in Wasser stattfindet, sind besonders diejenigen der Firma Becker und Ulmann in Berlin zu nennen. Es sind dies dieselben Apparate, welche zur Kochung von Speisen vielfach in Krankenhänsern, Kasernen, Gefängnissen und ähnlichen Anstalten Verwendung finden. Ihre Construction ist derart, dass ein mit fest verschliessendem Deckel versehener Kessel die zu kochenden Objecte aufnimmt, dessen Boden und Seitenwände von Dampf 
umspült werden. Der Dampf dient zur Erhitzung des im Kessel befindlichen, den Objecten zugesetzten Wassers.

Die Dampfkochapparate haben in ihrer Construction grösste Aehnlichkeit mit den Dampfdesinfectionsapparaten, wie sie zur Desinfection von Kleidern, Betten u. s. w. in Gebrauch sind. Sie stellen stehende oder liegende eiserne Cylinder dar, in welche das in Stücke zerlegte Fleisch in Metallkörben mit siebartig durchlöcherten Boden- und Seitenplatten eingebracht wird. Nach Beschickung der Apparate wird ihre Thür fest geschlossen. Dann wird entweder direct Dampf von einem Dampfkessel aus in die Apparate eingeleitet oder es wird durch Einleitung von hochgespanntem Dampf zwischen einen unter den Apparaten angebrachten Doppelboden auf ihrem Grunde stehendes Wasser in Dampf verwandelt, der die Luft aus dem Apparate verdrängt und das Fleisch umspült, durchdringt und gar kocht. Der Dampf kann in den Apparaten eine Druckhöhe bis zu 1 Atmosphäre Ueberdruck, entsprechend einer Temperatur von etwa $120^{\circ}$, erreichen. Die Grösse der Apparate ist so bemessen, dass sie das in Stücke zerlegte Fleisch von etwa einem bis drei Rindern oder mehreren Schweinen gleichzeitig aufnehmen können.

Die ersten Apparate dieser Art wurden vor etwa 8 Jahren von der Firma Dr. H. Rohrbeck in Berlin angefertigt. Die Rohrbeck'schen Apparate sind liegende Cylinder, bei denen die Kochung gewöhnlich durch directe Einleitung von Kesseldampf stattfindet, in denen aber auch eine Entwickelung von Dampf aus Wasser im Apparate selbst durch Heizung eines Doppelmantels mit Kesseldampf möglich ist. Die Apparate sind ausserdem mit Einrichtungen versehen, welche die Anwendung des von den sonstigen Desinfectionsapparaten Rohrbeck's her wohlbekannten Vacuumsystems mit Condensation und Druckdifferenzen ermöglichen. Am Boden des Apparates sammeln sich Condenswasser, ausgekochter Fleisehsaft und Fett und geben eine Fleischbrühe $a b$, die der Consument als Zugabe zu dem von ihm gekauften abgekochten Fleische erhält. Die Rohrbeck'schen Apparate haben grosse Verbreitung gefunden. In Folge der durch Anbringung der Condensationsvorrichtungen gegebenen complicirten Construction ist aber ihr Preis ein ziemlich hoher und ihre Bedienung eine nicht ganz einfache. Daher haben ihnen in den letzten Jahren weniger complicirt gebante billigere Apparate, und zwar namentlich die von den Firmen Rietschel und Henneberg-Dresden und Rud. A. Hartmann-Berlin construirten erhebliche Concurrenz gemacht. Die Apparate beider Firmen sind sich im Princip gleich. Die Dampfbildung erfolgt bei ihnen durch Erhitzung eines in den Apparat eingebrachten Wasserquantums mittels Kesseldampfes, der in den doppelten Boden des Apparates eingeleitet wird. Das in den Apparat eingefüllte 
Wasser verwandelt sich während des Kochprocesses in eine vortreffliche Fleischbrühe. Beide Apparate können auch zum Ausschmelzen von Fett benutzt werden. Sie unterscheiden sich von einander im Wesentlichen dadurch, dass der Rietschel-Henneberg'sche Apparat einen stehenden Cylinder, der Hartmann'sche einen liegenden Cylinder darstellt. Diese Verschiedenheit der Construction, die für den Kochprocess selbst wohl als irrelevant gelten kann, bringt es mit sich, dass der Henneberg'sche Apparat schwerer zu beschicken und zu entladen ist, da es ziemliche Mühe macht, die schweren, mit Fleisch gefüllten Körbe auf den Apparat zu heben, in inn herabzulassen und nach der Kochung wieder aus ihm emporzuziehen. Leichter beschickt sich der Hartmann'sche Apparat, in den die Körbe auf Rosten hineingeschoben werden, deren grössere Exemplare übrigens auch wie viele Dampfdesinfectionsapparate für Betten und dergl. einen herausziehbaren Wagen zum bequemen Ein- und Auspacken der Fleischkörbe besitzen.

Andere Dampfkochapparate, wie die ron Lümkemann-Budenberg in Dortmund, von Seiffert u. A. construirten sind den zuletzt beschriebenen ähnlich, haben bislang aber anscheinend nur geringe Verbreitung gefunden.

Es liegt auf der Hand, dass die Dampfkochapparate schnellere und gründlichere Durchkochung von Fleischstücken erzielen müssen als die Wasserkochapparate. Sie sind so eingerichtet, dass sie mit gespanntem Dampf von einer Temperatur, die beträchtlich über der des kochenden Wassers liegt, arbeiten, daher mehr als die Wasserkochapparate leisten können. Andererseits haben sie den Nachtheil, dass in ihnen das Fleisch mehr an Gewicht verliert als bei der Kochung im Wasser. Dass man auch im Becker-Ulmann'schen Apparate eine gute Durchkochung nicht zu grosser Fleischstücke in nicht gar zu langer Kochdauer erreichen kann, ist durch Versuche erwiesen. Die Frage, ob man für die Kochung bedingt gesundheitsschädlichen Fleisches den Dampf- oder den Wasserkochapparaten den Vorzug geben soll, wird daher wesentlich davon abhängen, ob die pathogenen Lebewesen im Fleische, deren Vernichtung man anstrebt, schwer oder leicht abzutödten sind.

Es kann nun, ganz kurz zusammengestellt, Fleisch von folgender Beschaffenheit als bedingt gesundheitsschädlich, aber nach guter Durchkochung ohne Schaden geniessbar angesehen werden:

1. Das Fleich von leicht finnigen Thieren. Da wir in Deutschland eine einheitliche Regelung der Fleischbeschau nicht besitzen, so gehen in dieser wie in vielen anderen Fragen die Vorschriften in den einzelnen Bundesstaaten, Regierungsbezirken und Communen weit aus einander. So 
verbieten z. B. Hamburg und einige preussische Regierungsbezirke die Verwendung des Fleisches finniger Schweine zum menschlichen Genuss überhaupt, während überall sonst die Verwendung schwach finnigen Fleisches in gekochtem oder gepökeltem Zustande gestattet ist. In Sachsen ist es erlaubt, Rindfleisch, wenn bei sorgfältiger Suche nur eine Finne gefunden wird, roh auf der Freibank zu verkaufen; im Regierungsbezirke Oppeln ist dagegen durch besondere Bestimmung festgelegt, dass schon Fleisch mit einer Finne als der Kochung oder Pökelung bedürftig gelten soll.

2. Das Fleisch ron trichinösen Thieren, wenn die Parasiten nicht zu zahlreich sind und das Fleisch den Eindruck gesunden Fleisches macht. Vorläufig hat nur Sachsen eine derartige Bestimmung eingeführt, während anderen Ortes wohl Rüeksicht auf $\S 367$, Ziffer 7 des Strafgesetzbuches, worin der Verkauf trichinōsen Fleisches mit Strafe bedroht und dasselbe unter den Begriff „verdorbene Nahrungsmittel" subsumirt wird, den Erlass ähnlicher Vorschriften, wie sie für Sachsen gelten, verhindert hat.

3. Das Fleisch von tuberculösen Thieren mit anscheinend localer Tuberculose, wenn es zweifelhaft ist, ob nicht doch Generalisation vorliegt, also namentlich beim Vorhandensein tuberculöser Erweichungsherde, Cavernen und bei beginnenden Ernährungsstörungen. Ferner das Fleisch von Thieren mit frischer, generalisirter Tuberculose, wenn dieselbe auf die Eingeweide (Nieren, Milz) beschränkt ist.

Fleisch von Thieren mit zweifellos localer, aber ausgebreiteter Tuberculose und mit generalisirter, abgeheilter, lediglich auf die Eingeweide beschränkter Tuberculose kann auf der Freibank roh verkauft werden; bei Fehlen einer Freibank für rohes Fleisch aber kann es ebenfalls gekocht feilgeboten werden.

Fleisch von tuberculösen Thieren mit ausgesprochener Abmagerung, mit tuberculōsen Veränderungen in den Fleischlymphdrüsen, in Knochen und Muskeln oder mit den Erscheinungen kürzlich erfolgter Blutinfection ist wegen seiner starken pathologischen Veränderungen und der dadureh bedingten unappetitlichen Beschaffenheit zum menschlichen Genusse auch im gekochten Zustande untauglich.

Fleisch von nicht abgemagerten Thieren, die nur unerhebliche locale Tuberculose zeigen, darf nach Entfernung der tuberculösen Theile unbedenklich ohne Beschränkung zum freien Verkehr zugelassen werden.

Dass die Gefahr einer Tubereuloseinfection durch den Genuss des Fleisches selbst stark tuberculöser Thiere nicht erheblich ist, haben zahlreiche Thierversuche sehr wahrscheinlich gemacht. ${ }^{1}$ Immerhin ist es

: Siehe die Litteratur bei Ostertag, Handbuch der Fleischbeschau. Stuttgart 1895. 2. Aufl. S. 535. 
zweifellos richtiger, Fleisch, das lebende Tuberkelbacillen enthalten könnte - ausser durch Generalisation gelangen sie in's Fleisch auch mit dem Messer des Schlächters, das soeben tuberculöse Organe des Thieres durchtrennt, gleich darauf Muskeln desselben durchschneidet - ganz vom Consum fernzuhalten oder nur in gekochtem Zustande dem Publikum zu überlassen. Dass das Fleisch tuberculöser Thiere Tuberculin in nennenswerther Menge nicht enthält, wissen wir durch Versuche von Eber; dass sein Nährwerth kein herabgesetzter ist, durch Untersuchungen von $R$ u m pel, ${ }^{1}$ während aus Experimenten von Kutscher ${ }^{2}$ hervorgeht, dass es in höherem Grade der Darmfäulniss zugänglich ist, als das Fleisch normaler Thiere.

4. Das Fleisch von Thieren mit septischen und pyämischen Erkrankungen, soweit es nicht durch eine Beschaffenheit unappetitlich ist und soweit eine Infections- oder Intoxicationsgefahr bei Genuss desselben in gekochtem Zustande nicht mehr droht. Wie man durch die bakteriologische Untersuchung über diesen letzteren Punkt sich Klarheit verschaffen kann, lernt man aus einer sehr lehrreichen Arbeit von Basenau, ${ }^{3}$ auf welche dieserhalb verwiesen sein mag.

5. Das Fleisch von Thieren mit grösseren Mengen von Psorospermien, Muskelstrablenpilzen, Kalkconcrementen, multiplen Hämorrhagieen nicht septiseher Natur kann eventuell ebenfalls Gegenstand der Kochung werden, wenn man sich aus irgend einem Grunde scheut, es in rohem Zustande zum Consum zuzulassen, andererseits aber seine Beschaffenheit eine absolute Zurückweisung von Consum nicht nöthig macht.

6. Auch Fleisch von rothlauf-, seuche-oder pestkranken Schweinen könnte gelegentlich vielleicht dem Kochapparate zngewiesen werden, wenn auch meistens die Dinge so liegen, dass das Fleisch frisch erkrankter Thiere roh als minderwerthig auf der Freibank verkauft werden könnte, während in den späteren Stadien das Fleisch als ungeniessbar zu bezeichnen ist. Wo keine Freibank für rohes Fleisch existirt, wie z. B. in Berlin und Hamburg, würde man das Fleisch frisch erkrankter Thiere nach Kochung in den Verkehr bringen können.

Das Fleisch milzbrand-, rotz- und wuthkranker Thiere würde in gut gekochtem Zustande ohne Schaden genossen werden können. Seine Verwerthung für den menschlichen Consum verbietet sich aber wegen der bei der Zerlegung des Fleisches zu besorgenden Infectionsgefahr.

1 Rampel, Archiv für Hygiene. Bd. XXVI. S. 386.

2 Kutscher, Ebenda. Bd. XXVII. S. 34.

${ }^{3}$ Basenau, Ebenda. Bd. XXXII. S. 219. 
Aus dem Vorstehenden ergiebt sich, dass - abgesehen von den unter 5. erwähnten Fällen, in denen die Kochung überhaupt nur grösserer Vorsicht wegen erfolgt - es neben Finnen und Trichinen ausschliesslich Parasiten bakterieller Natur sind, die im Fleische vernichtet werden sollen. Unbedingt wird man zur Abtōātung von Bakterien den intensiver wirkenden Dampfapparaten vor den Wasserkochapparaten den Vorzug geben, desgleichen für die Vernichtung von Trichinen, da diese Temperaturen bis zu $70^{\circ}$ überdauern können. Für die Abtödtung von Finnen, die erfahrungsgemäss bei $5^{\circ}{ }^{\circ}$ stets schon sicher vernichtet werden, kann man dagegen, da diese Temperatur immer bei verständigem Betriebe der Wasserkochapparate im Innern nicht zu grosser Fleischstücke zu erreichen ist, die Wasserkochapparate heranziehen. Natürlich steht niehts im Wege, finniges Fleisch auch im Dampfapparate zu kochen, wenn ein Wasserkochapparat nicht vorhanden ist, oder durch Pökeln oder, wie es neuerdings in Aufnahme kommt, durch prolongirte Kühlung die Finnen in Fleische abzutödten.

Den hier gegebenen Ausführungen entsprechend hat man allgemein die Aufgabe der Wasser- und Dampf Kochapparate so umgrenzt, dass den ersteren die Kochung finnigen, den letzteren die Kochung mit Trichinen oder pathogenen Bakterien inficirten Fleisches zugewiesen wird.

II.

Als im Jahre 1897 die zuständige Behörde in Hamburg sich entschloss, Apparate zur Abkochung bedingt gesundheitsschädlichen Fleisches auf dem Schlachthofe aufzustellen, wandte sie sich an das staatliche hygienische Institut zu Hamburg mit dem Ansuchen, das Institut möge ein Gutachten darüber abgeben, welche der in Frage kommenden Apparate als die geeignetsten zur schnellen und sicheren Abtödtung der im Fleische enthaltenen pathogenen Lebewesen anzusehen seien. Speciell wurde, nachdem die sachverständigen Beamten der Schlachthofverwaltung und der Veterinärbehörde dahin neigten, einer Beschaffung von Apparaten der Firma Hartmann das Wort zu reden, ein Urtheil darüber gewünscht, ob die Fleischkochapparate Hartmann'scher Construction oder die von manchen Seiten als besonders empfehlenswerth bezeichneten Apparate der Firma Rohrbeck als gleichwerthig anzusehen seien oder ob einer derselben den Vorzug verdiene. Des weiteren wurde um eine Aeusserung darüber ersucht, ob gegen die Anwendung eines Becker-Ulmann'schen Wasserkochapparates für die Sterilisirung leicht finnigen Schweinefleisches sanitäre Bedenken geltend zu machen seien. 
Beauftragt, das Gutachten über diese Fragen zu erstatten, erklärte ich den oben gemachten Ausführungen entsprechend, dass die Verwendung Becker-Ulmann'scher Apparate zur Kochung finnigen Fleisches als geeignet anzusehen sei. Was die Frage anbelangte, ob für die Kochung von bedingt gesundheitsschädlichem Fleische anderer Art der Ro hrb eck'sche oder der Hartmann'sche Apparat den Vorzug verdiene oder ob beide gleichwerthig seien, so glaubte ich mich in dem Sinne äussern zu müssen, dass beide Apparate wesentlich dasselbe leisten würden. Die Behörde entschied sich darauf für die Anschaffung zweier grosser Hartmann'scher Dampfkochapparate von 2100 bezw. 2700 Liter Fassungsvermögen.

Zur Begründung der von mir geäusserten Ansicht mögen folgende, dem Gutachten entnommene Passus hier Platz finden:

Die Rohrbeck'sehe Desinfectionsmethode hat folgende Eigenheiten: Nachdem zunächst durch längeres Durchblasen von Dampf durch den Apparat die denselben füllende Luft möglichst gut entfernt ist, wird die Dampfausströmungsöffnung geschlossen, darauf, nachdem ein mässiger Ueberdruck im Innern des Apparates entstanden ist, auch die Dampfzuleitung abgesperrt. Nunmehr wird plötzlich, durch Füllen des Zwischenraumes zwischen beiden Apparatmänteln mit kaltem Wasser, die Temperatur des Apparates stark herabgesetzt, der in ihm enthaltene Dampf zur Condensation gebracht und ein Vacuum im Apparate erzeugt. Nachdem dies eingetreten, wird auf's Neue der Dampfzufluss geöffnet und von Zeit zu Zeit während des Kochprocesses eine Kühlung und Vacuumbildung in der geschilderten Weise ausgeführt.

Von dieser Kühlung des Apparates, welche auf den ersten Blick ganz unzweckmässig erscheinen könnte, da sie ja die Temperatur des Apparates herabsetzt, anstatt. sie dem gewünschten Siedepunkte zu nähern, verspricht sich Rohrbeck zweierlei Vortheile: Erstens nämlich soll beim Eintritt des Vacuums eine Verdünnung der Luft, welche in den Poren der zu desinficirenden Objecte enthalten ist und die Durchdringung derselben mit dem am besten desinfectorisch wirksamen gesättigten Dampfe inhibirt, entstehen. Wenn dann durch die folgende Oeffnung des Dampfzuflusses wiederum normaler Druck und Ueberdruck eintritt, sollen die Poren der Objecte sich besser mit Dampf füllen. Der wiederholte Wechsel zwischen Vacuum und Ueberdruck soll schliesslich eine vollständige Entfernung der Luft aus den Objecten und einen Ersatz derselben durch gesättigten Dampf gewährleisten.

Zweitens dient nach Robrbeck die bei der Condensation des Dampfes zu Wasser verfügbar und frei werdende Verdampfungswärme dazu, die Objecte stark zu erwärmen, so dass deren Temperatur in Folge der Abkühlung des Apparates nicht etwa sinkt, sondern steigt. Auf diese Weise 
soll die Temperatur von $100^{\circ} \mathrm{C}$., welche zu vollkommener Durchdämpfung der Objecte nöthig ist, leichter in ihrem Innern erreicht werden.

Bei der Würdigung dieser beiden, von Rohrbeck behaupteten Vorzüge seiner Desinfectionsmethode ist zu berücksichtigen, dass dieselben bei der Desinfection von Fleisch nicht ganz so zutreffen, wie bei der Desinfection von Betten, Wäsche, Kleidern und dergleichen, wofür die Rohrbeck'schen Apparate ursprünglich bestimmt worden sind. Die genannten Erzeugnisse der Textilindustrie, wie auch die mit locker gepackten Federn, Seegras- oder Strohmassen ausgestopften Betten enthalten in reichlicher Menge mit Luft gefüllte Maschen und Hohlräume. Aus ihnen lässt sich die Luft durch die Einwirkung von Dampf natürlich nur allmählich und vielleicht schneller herausdrängen, wenn durch Erzeugung eines Vacuums die Luft direct aus den Objecten herausgesaugt wird. Anders ist es beim Fleische. Die Structur desselben, und zwar sowohl diejenige des Muskelgewebes, als auch die des Binde- und Fettgewebes schliesst es aus, dass grössere oder auch nur nennenswerthe Mengen von Luft sich in ihm befinden. Besondere Maassnahmen zu ihrer Entfernung erübrigen sich demnach, und zwar um so mehr, als bei der allmählichen Temperatursteigerung im Fleische, welche durch Leitung der Wärme in der Fleischsubstanz ohne Beeinträchtigung durch die eingeschlossenen Luftmengen stattfindet, eine Dampfentwickelung von dem in Fleische selbst enthaltenen Wasser ausgehen muss; die Luft wird dann nicht durch von aussen eintretenden Dampf, sondern durch den im Object selbst gebildeten hinausgedrückt.

Während dem zu Folge der erste von Rohrbeck seinem Verfahren nachgerühmte Vortheil für die Desinfection von Fleisch nicht in Betracht kommt, ist die Bedeutung des zweiten Factors dieselbe wie bei der Desinfection von Betten und dergleichen. Es ist nun hierbei zu bemerken, dass eine Einigung darüber, ob die Rohrbeck'sche Condensation wirklich ein schnelleres Eindringen der Hitze in das Innere der Objecte ermöglicht, noch keineswegs erzielt ist. Die mehrfach von Rohrbeck verfochtene Zweckmässigkeit seiner Condensation hat von anderer, ebenfalls als sachverständig za bezeichnender Seite lebhaftesten Widerspruch erfahren. Ueberlegt man ohne Voreingenommenheit die Verhältnisse, so wird man sich sagen müssen, dass die Condensation des Dampfes im Apparate rorzugsweise an den Stellen erfolgen muss, wo die Abküblung am stärksten wird, d. h. aber, da die Kühlung von aussen her erfolgt, an den Wandungen der Apparate, nicht auf den Objecten. Die bei der Dumpfcondensation frei werdende Wärme wird daher zur Erhitzung der Apparatwände dienen, von diesen aber auf das sie umspülende kalte Wasser übergehen. Tritt nun wieder Dampf in den Apparat ein, so muss ein ganz bedeutendes Quantum desselben nothwendig sein, um erst wieder 
die vor der Abküblung erreichte Temperatur herzustellen. Man kann sich ja wohl denken, dass so wie die Wände auch die Objecte im Innern des Apparates in Folge der Dampfcondensation eine Temperaturerhöhung erfahren können; aber dieselbe wird gering bleiben, weil die Objecte nicht die kältesten Stellen im Apparate sind, folglich auch nur ein geringer Theil des Dampfes auf ihnen sich verdichten wird. Directe Versuche über die Erhitzung der Objecte mit Hülfe von Vacuumbenutzung haben Rohrbeck gute Resultate geliefert, anderen Untersuchern, wie Sander und Clarenbach, ${ }^{1}$ keine Vortheile dargethan, insofern sie mit oder ohne Benutzung der Luftleere die gewünsehte Temperatur im Innern der Objecte in gleich langer Zeit erzielten. Man kann also nicht sagen, dass mit unbezweifelbarer Gewissheit der von Rohrbeck behauptete Vortheil schneller Objecterhitzung bei Anwendung seines Kühlverfahrens dargethan ist.

Hier hätte in das Gutachten noch eingefügt werden können, dass es nicht unbekannt ist, dass im Innern bestimmter Objecte auch bei Erhitzung im strömenden Dampf von $100^{\circ}$ Temperaturen entstehen können, die weit über $100^{\circ}$ liegen; dass daher die von Rohrbeck bei seinem Vacuumsystem im Inners von Objecten beobachteten hohen Temperaturen nicht nothwendig Resultat der besonderen Methode der Desinfection sein müssen. Auf die Erklärung dieser paradox erscheinenden Thatsache soll hier nicht näher eingegangen, bezüglich derselben vielmehr auf eine kürzlich erschienene Arbeit von Rubner ${ }^{2}$ verwiesen werden. Es ist daher zu erwarten, fährt das Gutachten fort, dass mit Apparaten, welche ähnlich wie der Rohrbeck'sche Desinfector construirt sind, aber ohne die specifische Rohrbeck'sche Desinfectionsmethode arbeiten, principiell das Gleiche wie mit diesem zu erreichen sein wird. So z. B. mit dem Hartmann'sehen Dampfapparate.

Des Weiteren wird ausgeführt, dass in der Litteratur genaue Protocolle über Experimente mit diesem Apparate, die allen zu berücksichtigenden Punkten, wie Abtödtung der pathogenen Organismen, Zeitdauer der Sterilisation, Höhe der erreichten Temperatur u. s. w., Aufmerksamkeit zugewendet hätten, nicht vorliegen. Indessen sei auch ohne derartige Angaben die Gleichwerthigkeit der Hartmann'schen mit den Rohrbeck'schen Apparaten aus verschiedenen Gründen zu folgern. Einmal hatte ein Schlachthofdirector, der früher mit einem Rohrbeck'schen Apparate gearbeitet hatte, sich dahin ausgesprochen, dass der von ihm späterhin benutzte Hartmann'sche Kochapparat eben so sicher und schnell wie der Rohrbeck'sche arbeite, dabei leichter zu bedienen sei und das Fleisch

\footnotetext{
I Sander u. Clarenbach, Gesundheits-Ingenieur. 1893. S. 642.

${ }^{2} \mathrm{Rubner}$, Hygienische Rundschau. 1898. Nr. 15. S. 721.
} 
weniger stark zum Zerfalle bringe. Zweitens hatten Versuche von Rieck ${ }^{1}$ und Liebe ${ }^{2}$ eine gute Function des Rietschel-Henneberg'schen Kochapparates, der, wie obèn gezeigt, dem Hartmann'schen im Princip gleich ist, woraus zu folgern sein dürfte, dass auch die Hartmann'sche Construction den $\mathrm{zu}$ stellenden Anforderungen entspricht. Endlich konnte auch aus der Thatsache, dass in den letzten Jahren viel mehr Communen den Hartmann'schen als den Rohrbeck'schen Apparat einführen, gefolgert werden, dass beide gleich viel leisten; ohne Zweifel werden die betreffenden Städteverwaltungen sich nicht für den einen oder den anderen Apparat entschieden haben, ohne vorher das Urtheil Sachverständiger einzuholen.

Auf Grund der erwähnten Thatsachen und Erwägungen musste man zu dem Resultate kommen, dass speciell für die Zwecke der Fleischabkochung der Rohrbeck'sche Apparat keine wesentlichen Vortheile gegenüber dem Hartmann'schen Apparate, was die Sicherheit und auch die Schnelligkeit der Sterilisation anbetrifft, biete. Es konnte darauf hingewiesen werden, dass es bei der Einrichtung der Hamburger Desinfectionsanstalten auch nicht als nöthig erachtet worden war, nach den Rohrbeck'schen Principien arbeitende Apparate aufzustellen, trotzdem, wie oben dargelegt, für die dort auszuführende Desinfection von Kleidern, Betten und dergleichen noch eher als für die Fleisehdesinfection die Rohrbeck'sche Methode Bedeutung beanspruchen könnte.

Am Schlusse des Gutachtens erklärte ich es für zweckmässig, dass dem hygienischen Institute Gelegenheit geboten werde, durch Versuche die Brauchbarkeit der Hartmann'schen Apparate festzustellen und eine Methode der Kochung ausfindig zu machen, welche sichere Abtödtung der pathogenen Keime im Fleische gewährleiste ohne zu starke Beeinträchtigung der Beschaffenheit des Fleisches als Nahrungsmittel zu bedingen. Diesem Ansuchen entsprechend, stellte die Schlachthof behörde in entgegenkommendster Weise dem hygienischen Institute die Kochapparate und Fleisch confiseirter Thiere zu Versuchen zur Verfügung, über deren Ergebnjsse im Folgenden berichtet werden soll.

\section{III.}

Bei dem im Auftrage des Institutes ausgeführten Versuchen glaubte ich mich von folgenden Ueberlegungen leiten lassen zu sollen:

Ein Kochapparat für bedingt gesundheitsschädliches Fleisch hat zwei Bedingungen zu erfüllen, nämlich:

${ }^{1}$ Rieck, Archiv für wissenschaftl. u. prakt. Thierheilkunde. Bd. XXI.

${ }^{2}$ Liebe, Zeitschrift für Fleisch- und Milchhygiene. 1894. Bd. IV. S. 143. Zeitschr. P. Hygiene. $\mathbf{X X X}$. 
1. bei sachgemässem Betrieb eine sichere Abtōdtung aller im Fleische vorhandenen pathogenen Lebewesen, deren Gegenwart die Gesundheitsschädlichkeit des Fleisches eben bedingt, zu gewährleisten, und

2. das Fleisch gleichzeitig in einem Zustande zu liefern, in dem es quoad Appetitlichkeit, Geschmack und Nährwerth als gutes Nahrungsmittel $\mathrm{zu}$ bezeichnen ist.

Beide Ziele gemeinsam in rationeller Weise zu erreichen, ist die einzige Schwierigkeit beim Betriebe der Dampf-Fleisch-Kochapparate. Dass man bei genügend langer Dampfwirkung jede beliebige Art von Organismen in jedem beliebig grossen Stücke Fleiseh abtödten kann, ist zweifellos. Die Frage ist nur, ob das Fleisch schliesslich noch geniessbar ist. Gar zu lange wird man Fleisch, das Genusszwecken dienen soll, nicht gern kochen. Je länger es kocht, desto mehr verliert es an Gewicht. Mit der Dauer des Kochens verliert es in Folge des Auspressens und Auslaugens der Fleischsalze aber auch immer mehr an Schmackhaftigkeit, die ihm durch Zugabe der die Salze enthaltenden Bouillon an den Käufer doch nicht wieder gegeben werden kann. Daher ist nicht nur vom Standpunkte des Verkäufers, der möglichst viel Nutzen aus dem Verkaufe des Fleisches ziehen und daher gar zu erhebliche Gewichtsverluste desselben in Folge der Kochung vermieden wissen will, und nicht nur im Interesse einer regeren Nachfrage nach dem Fleische, die sich bei guter Beschaffenheit desselben natürlich steigern wird, sondern auch im Sinne des armen Consumenten, der für sein schwer erworbenes Geld auch möglichst gute Waare verlangen kann, eine Beschränkung der Kochzeit auf diejenige Dauer wünschenswerth, die gerade zu gutem Garkochen des Fleisches genügt, - vorausgesetzt natürlich, dass es gelingt, das Heisch dabei auch seiner infectiösen Eigenschaften zu entkleiden.

Man hat allgemein als principielle Bedingung für die Ausführung der Kochung bedingt gesundheitsschädlichen Fleisches gefordert, dass im Innern, d. h. an den für die Hitze am schwersten zugänglichen Stellen der zu kochenden Fleischstücke eine Temperatur von $100^{\circ}$ erreicht sein muss. ${ }^{1}$ $\mathrm{Ja}$ das sächsische Ministerium des Innern hat sogar verlangt, ${ }^{2}$ dass die Temperatur vou $100^{\circ}$ etwa eine halbe Stunde einwirken soll, ehe die Kochung als vollendet gelten und der Apparat geöffnet werden darf. Man

1 Vgl. Hertwig, Deutsche Vierteljahresschrift für öffentl. Gesundheitsplege. Bd. XXIV. - Maske, Zeitschrift für Fleisch- und Milchhygiene. Jahrg. II. 1893. S. 115. - Rieck, Archiv f. wissensehaftl. u. prakt. Thierheilleunde. Bd. XXI u. s. w.

V Verordnung vom 17. Decbr. 1892. Anweisung für die Ausführung derselben $\S 4$ b) aa) (Veröffentl. des Kaiserl. Gesundheitsamtes, 1893, S. 356). Eine gleichlautende Bestimmung gilt für Anhalt. (Ebenda, 1896, S.694.) 
weiss, dass in Dampf von $100^{\circ}$ alle uns bekannten pathogenen Mikroorganismen, auch die Sporen des Milzbrandbacillus, in kurzer Frist, in wenigen Minuten höchstens, zu Grunde gehen, dass daher ein Object, welches in allen seinen Theilen einige Minuten lang die Einwirkung feuchter Hitze von $100^{\circ}$ erfahren hat, frei von Infectionserregern sein muss. Die Forderung, dass im Innern der Fleischstücke $100^{\circ}$ erreicht sein sollen, hat dem zu Folge eine durchaus berechtigte Grundlage. Es fragt sich nur, ob es nöthig ist, dass im Fleische, um dasselbe von pathogenen Lebewesen $\mathrm{zu}$ befreien, die Temperatur von $100^{\circ}$ erreicht wird und ferner, ob es zweckmässig ist, die Vorschriften für die Kochung so zu formuliren, dass die Dauer der Kochung davon abhängig gemacht wird, wann die Temperatur von $100^{\circ}$ im Fleischinnern erreicht worden ist. Es ist hier Folgendes zu bedenken:

1. Die pathogenen Organismen, welche wir im Fleische abtödten wollen, werden schon bei Temperaturen weit unter $100^{\circ}$ - immer die Einwirkung feuchter Hitze vorausgesetzt - in kurzer Frist und mit Sicherheit vernichtet.

Dem Fleischkochapparate werden zur Unschädlichmachung hauptsächlich Thiere mit ausgebreiteter oder generalisirter Tuberculose zugewiesen. In Hamburg sind bis heute bis auf zwei oder drei Ausnahmen sogar ausschliesslich tuberculöse Thiere in der Fleischkochanstalt verarbeitet worden. Durch exact ausgeführte Versuche von Yersin ${ }^{1}$, Forster, ${ }^{2}$ de Man ${ }^{3}$ und Bonhoff ist nun festgestellt worden, dass Erhitzen tabereulöser Materialien während 20 Minuten bis 1 Stunde auf $60^{\circ}$, während 10 Minuten auf $70^{\circ}$, während 5 Minuten auf $80^{\circ}$ und momentan auf $85^{\circ}$ genügt, um die Tuberkelbacillen in ihnen abzutödten. Zum Unschädlichmachen tuberculösen Fleisches ist eine Temperatur von $100^{\circ}$ also absolut nicht von Nöthen, vielmehr eine Temperatur von 70 bis $80^{\circ}$ bei etwa 5 : Minuten langer Einwirkungsdauer vollkommen ausreichend.

Neben tuberculösem Fleische kommt nach dem oben Erörterten für die Kochapparate noch in Betracht das Fleisch von Schlachtthieren, welche an pyämischen oder septicämischen Affectionen gelitten baben, soweit es nicht wegen unappetitlicher Beschaffenheit als ungeniessbar zu beseitigen ist und soweit der Verdacht vorliegt, dass sein Genuss in rohem oder mangelhaft gekochtem Zustande infectiös oder toxisch wirken kann ${ }^{5}$, ferner

1 Yersin, Annales de l’Institut Pasteur. T. II. p. 60.

${ }^{2}$ Forster, Hygienische Rundschau. Bd. II. Nr.20. S. 869.

3 de Man, Archiv für Hygiene. Bd. XVIII. S. 133.

4 Bonhoff, Hygienische Rundschau. Bd. II. Nr. 23. S. 1009.

- Vgl. hierza die Arbeit von Basenau, Archiv f. Hygiene. Bd. XXXII. S. 219. 
eventuell das Fleisch von pest-, seuche-, und rothlaufkranken Schweinen und endlich das Fleisch von Thieren mit Trichinen, Finnen und sehr zahlreichen Psorospermien. Die Trichinen und Finnen - über die für den Menschen kaum schädlichen Psorospermien ist in dieser Hinsieht nichts bekannt - und die Erreger der vorerwähnten Infectionskrankheiten sind alle, zahlreichen Experimenten zu Folge, durch Temperaturen von etwa $70^{\circ}$ fast momentan abzutödten,$^{1}$ also noch weniger resistent als die Tuberkelbacillen, so dass auch zu ihrer Vernichtnng die Erreichung einer Temperatur von $100^{\circ}$ keineswegs erforderlich ist.

2. Dass es nicht nöthig ist, um Fleischstũcke gut gar zu kochen, in ihnen eine Temperatur von auch nur annähernd $100^{\circ}$ zu erzielen, ist eine so bekannte Thatsache, dass es nicht nöthig ist, hier weiter darauf einzugehen. Es sei nur daran erinnert, dass beim Kochen von Fleisch im Haushalt die Temperatur im Fleischinnern fast stets und oft sehr tief unter $100^{\circ}$ bleibt und dass das Fleisch dabei durchweg gar und zart sein kann. ${ }^{2}$ Auch nach dieser Richtung liegt also kein Grund vor, eine Temperatur von $100^{\circ} \mathrm{im}$ Innern der zu kochenden Fleischstücke zu verlangen.

3. Um zu erfahren, wann im Innern der im Kochapparat befindlichen Fleischstücke die Temperatur von $100^{\circ}$ erreicht ist, bringt man in die Stücke Contactapparate hinein, welche, sobald sie auf $100^{\circ}$ erwärmt worden sind, ein ausserhalb des Kochapparates befindliches elektrisches Läutewerk in Gang setzen. Solche Contactapparate sind theuer; ihre Verbindung mit den Läutewerken erfordert die Anlage besonderer Einrichtungen am Kochapparat. Man kann daher der Regel nach nur ein, zwei oder höchstens drei Fleischstücke mit solchen Apparaten versehen und erfährt daher auch nur, wann in diesen Stücken die Temperatur von $100^{\circ}$ erreicht ist. Es ist durchaus nicht gesagt, dass dann auch in den anderen Stücken schon die Temperatur von $100^{\circ}$ überall herrscht. Der Einwand, dass man ja die augenscheinlich am schwersten permeablen Fleischstücke mit $100^{\circ}$ Läutewerken armiren kann, so dass zweifellos, wenn in ihnen $100^{\circ}$ herrschen, auch in den anderen Stücken $100^{\circ}$ erzielt sind, ist hinfällig, weil der Augenschein nach dieser Hinsicht, wie die Erfahrung lehrt, trügen

${ }^{1}$ S. bezügl. der Trichinen Leuckart, Untersuchungen über Trichina spiralis. 2. Aufl. Leipzig 1866. - Betreffs der Finnen Hertwig, Zeitschrift für Fleisch-u. Milchhygiene. Bd. II. S. 212, Ref. - Hinsichtlich der Bakterien z. B. van Geuns, Archiv für Hygiene. Bd. IX. S. 369 und die Lehrbücher der Bakteriologie.

2 Vgl. die Versuche von Iiiebig, Chem. Briefe. 1851. S. 503. - Wolffhügel u. Hüppe, Mittheilungen aus dem Kaiserl. Gesundheitsamte. Bd. I. S. 395. - Perroucito, cit. Ebenda. - Petri. Ebenda. Bd. VI. S. 266. - Fiore, Ann. d'Igiene speriment. 1897. S. 21. - Vallin, Revue d'Hygiène. 1897. S. 800 a. A. 
kann, und manchmal kleine, anscheinend leicht zu durchkochende Stücke dem Eindringen der Hitze mehr Widerstand bieten, als grosse, aller Vermuthung nach schwer bis in's Innerste zu erwärmende. Sehr wesentlich ist in dieser Beziehung, dass die Fleischstücke beim Erwärmen in Folge des Austretens von Saft und der aus der Eiweisscoagulation resultirenden Zusammenziehung der Muskelsubstanz unvorhersehbare. Veränderungen der Form und Beschaffenheit erleiden, die in einem Falle zur Bildung eines festen compakten Klumpens, im anderen zur Entstehung kleinerer, von Rissen und Fugen durchzogener Muskelballen führen. Die dieser Arbeit beigefügten Versuchsprotocolle lassen deutlieh erkennen - wie weiter unten noch näher dargelegt werden wird -, dass in zwei annähernd gleich beschaffenen Stücken die Hitze ganz verschieden sein kann; sie zeigen auch (Versuchsreihe I und II), dass noch nicht sicher überall in einem Stück $100^{\circ}$ erreicht sind, wenn ein in dessen Centrum eingeführter Contactapparat $100^{\circ}$ meldet.

Will man aber, wie der Erlass des sächsischen Ministeriums, die Ungewissheit darüber, ob zur Zeit, wann in ein em Stück $100^{\circ}$ erreicht sind, auch in den anderen $100^{\circ}$ herrschen, dadurch wett machen, dass man noch eine halbe Stunde weiter erhitzt, nachdem das erste Fleischstūck $100^{\circ}$ signalisirt hat, so wird man dureh dies prolongirte Kochen, selbst wenn der $100^{\circ}$-Apparat in einem der kleinsten Stücke lag, eine Anzahl der Fleisehstücke mit Sicherheit zu stark auskochen, so dass sie mürbe werden, zerfallen und damit sowohl schwer verkăuflich, als auch zu einem weniger guten Nahrungsmittel werden.

4. Die Apparate, welche bestimmt sind, das Ansteigen der Temperatur im Fleischinnern auf $100^{\circ} \mathrm{zu}$ melden, sind recht ingeniös construirte, im Ganzen richtig functionirende, aber auch sehr complicirte und fragile Gebilde. ${ }^{1}$ Schon an ganz neuen Apparaten erlebt man es nicht

${ }^{1}$ Für Fleischstücke ist ein besonderes Contact-Pyrometer von Duncker constrairt worden. (Abgebildet in Weyl's Handbueh der Hygiene, Bd. III, S. 447.) Eine Spiralfeder wird durch ein bei $100^{\circ}$ schmelzendes Metalllegierungsplättchen gespannt; sobald das Plättchen erweicht, durchbohrt die Feder dasselbe und schliesst einen elektrischen Contact. Brauchbar erwiesen sich mir auch Contactthermometer von Stuhl-Lautenschläger (Katalog Nr.60 des Letzteren, S. 34) u. von Muencke (unter der Bezeichnung: Elektrische 'Thermometer-Contactvorrichtung). Der Lauten schläger'sche Apparat lässt sich so einstellen, dass er Temperaturen zwischen 85 und $125^{\circ}$ meldet, der Muencke'sche so, dass er bei Temperaturen zwischen 10 und $360^{\circ}$ läutet; bei beiden Apparaten kann man auch feststellen', welches die höchste Temperatur war, der sie ausgesetzt gewesen sind. Für Fleischkochversuche nicht brauchbar sind die Kapselapparate nach Wolff-Merke und ähnliche Apparate, weil sie zu stark verschmutzen. 
selten, dass sie aus diesem oder jenem Grunde versagen. Dann steht der den Apparat Bedienende vor dem Kessel und wartet und wartet auf das Läutesignal, bis ihm schliesslich die Geduld ausgeht und er beim Oeffnen des Kessels den Grund für das Versagen des Läuteapparates, der lāngst hätte signalisiren müssen, findet. Inzwischen ist das Fleisch durch das zu lange Kochen aber wesentlich verschlechtert worden!

Ausserdem befördert der wiederholte Aufenthalt der Läuteapparate im Kessel, ihre dabei erfolgende Verschmutzung mit Fett und Fleischsaft ihre Haltbarkeit nicht eben. So sind z. B. die von Duncker speciell für Temperaturmessungen im Fleisch construirten Lälteapparate schon nach wenigen Kochungen kaum noch wieder in brauchbaren Zustand zu versetzen. Andere Läuteapparate sind so zerbrechlich, dass ihnen schon dadurch nur eine kurze Lebensdauer beschieden ist.

So macht man denn die Erfahrung, dass in der ersten Zeit des Betriebes eines Fleischkochapparates wohl der $100^{\circ}$ meldende Contactapparat jedes $\mathrm{Mal}$ in Gebrauch genommen wird, dass er aber bald fortgelassen und sein Läuten durch die Lehren der Erfahrung ersetzt wird. Der Leiter eines Fleischkochbetriebes kümmert sich dann nicht mehr darum, ob das Innere der Fleischstücke die Temperatur von $100^{\circ}$ erreicht, sondern er kocht eine ihm angemessen erscheinende Zeit hindurch und überzeugt sich schliesslich, ob auch die dicksten Stücke im Innern völlig gar gekocht sind.

Die Ersetzung der Contactapparate durch Maximalthermometer ändert an der Sachlage nicht viel. Bei ihrer Anwendung bekommt man kein Signal, wenn $100^{\circ}$ im Fleischinnern erreicht sind. Bemerkt man nach beendeter Kochung aber, dass die verwendeten Maximalthermometer $100^{\circ}$ und darüber zeigen, so hat man anch noch keine Gewissheit, dass in den nicht mit Thermometern versehenen Stücken die Temperatur $100^{\circ} \mathrm{er}$ reicht hat.

Die vorstehend skizzirten Ueberlegungen führen zu dem Schlusse, dass es einerseits nicht nöthig ist, zu verlangen, dass die Fleischstücke in den Fleischkochapparaten die Temperatur von $100^{\circ}$ in allen ihren Theilen erreichen, da sowohl eine Abtödtung der in Frage kommenden pathogenen Lebewesen im Fleisch schon bei Temperaturen weit unter $100^{\circ}$ sicher erfolgt, als auch ein Garkochen des Fleisches schon bei weniger als $100^{\circ}$ vollkommen gelingt. Sie zeigen aber andererseits auch, dass es nicht zweckmässig ist, als Merkmal für die Ausführung der Kochung die Erreichung einer Temperatur von $100^{\circ}$ in einem oder mehreren Fleischstücken zu wählen. Denn die Messung von $100^{\circ}$ in einem oder in einigen Stücken Fleisch giebt noch nicht die Gewähr, dass in den anderen eine annähernd gleich hohe Temperatur herrschto Will man dieser Unsicher- 
heit dadurch begegnen, dass man nach der Vorschrift des sächsischen Ministeriums noch eine halbe Stunde lang weiter kocht, nachdem das Contactthermometer in einem Stück $100^{\circ}$ gemeldet hat, so läuft man Gefahr, manche Stũcke zu lange zu kochen, so dass ihre Beschaffenheit zu ihrem Nachtheil verändert wird. Ferner aber sind die $100^{\circ}$-Läuteapparate oft unsicher in ihrer Function, verderben oder zerbrechen bald, machen dadurch erhebliche Kosten für Reparatur und Neuanschaffung und werden, wenn nicht beständige Beaufsichtigung der Fleischkochungen statt hat, daher bald überhaupt nicht mehr zur Controle der Kochung herangezogen, worauf einfach empirisch gearbeitet wird, ohne dass eine sichere Unterlage für die empirische Ausführung der Kochung vorhanden ist.

Man könnte des Weiteren gegen die $100^{\circ}$-Mlethode auch noch den Grund in's Feld führen, dass die Anzeige einer Temperatur von $100^{\circ}$ im Fleische noch nichts darüber aussagt, ob es auch feuchte Hitze von $100^{\circ}$ ist, die einwirkt; es könnte sein, dass mit Luft gemischter, nicht gesättigter Dampf von $100^{\circ}$ an der Lagerstelle des Thermometers vorhanden ist. Auf diesen Grund möchte ich nicht viel Gewicht legen. Mag es auch sein, dass die Beimischung von Luft zum Dampf dessen Desinfectionskraft herabsetzt, so wird dieser Factor doch kaum in Rechnung zu stellen sein, wenn man von vornherein bei der Ausführung der Kochung für möglichst vollständige Vertreibung der Luft aus dem Apparate sorgt.

Die Erzielung von $100^{\circ}$ im Innern der Fleischstücke hat man darum gefordert, weil man glaubte, dann der Abtödtung aller pathogenen Lebewesen im Fleisch sicher zu sein. Der nicht unwichtigen öconomischen und auch hygienischen Frage, ob das dabei gewonnene Kochproduct auch in seiner Qualität das bestmögliche zu erhaltende ist, hat man gar keine oder nur ganz nebensächliche Bedeutung beigelegt. Aus dem im Vorstehenden Erörterten geht klar hervor, dass kein zwingender Grund vorliegt, vielmehr manches dagegen spricht, das Erreichen einer Temperatur von $100^{\circ}$ im Fleischinnern, wie es in den bisher bekannt gewordenen Versuchsreihen der Fall gewesen ist, als Ziel und Zweek der Kochung und als Grundlage für eine etwa zu gebende Betriebsinstruction anzusehen. Als Richtschnur für Versuche, welehe festlegen sollen, wie man mit einem Fleischkochapparat am zweckmässigsten arbeitet, muss die Feststellung dienen, durch welche Art der Kochung die pathogenen Organismen des Fleisches sicher abgetödtet werden, während gleichzeitig das Fleisch gut gargekocht, saftig und wohlschmeckend wird, bezw. bleibt. Gelingt es, durch Versuche dieser Art eine sichere Basis für eine Kochvorschrift zu finden, so kann es gleichgültig bleiben, ob dabei $100^{\circ}$ im Fleisehinnern erreicht werden oder nicht. 
IV.

Nach Maassgabe dieser Gesichtspunkte wurden die in den Tabellen am Schluss dieser Arbeit wiedergegebenen Versuche III bis XXV unternommen.

Zuvor wurde bei 19 Kochungen, die zum Zwecke des Verkaufes von Fleisch in der Kochanstalt vorgenommen wurden, mit Läutewerken und Maximalthermometern in einzelnen Fleischstücken die erzielte Temperatur gemessen und dabei festgestellt, dass man besonders bei Stücken von nicht mehr als 2000 bis $3000 \mathrm{grm}$ Schwere durch 2- bis 21/2 stündiges Kochen Temperaturen von $100^{\circ}$ oder wenigen Graden darunter im Innern derselben erreichen, mithin mit den Hartmann'schen Apparaten etwa dasselbe leisten kann, wie mit den von Rohrbeck und von Rietschel und Henneberg construirten, über deren Functionen genaue Versuchsprotocolle in der Litteratur sich finden. ${ }^{1}$ Die Ergebnisse aus diesen 19 Experimenten sind in Versuchsreihe I und II zusammengestellt.

In den Versuchen III bis XXV wurden mit pathogenen Bakterien inficirte Fleischstücke der Kochung in den Apparaten unterzogen.

Mit Tuberkelbacillen wurde in allen Versuchen experimentirt, weil eben das Abtödten dieser Bakterien die häufigste und schon darum wichtigste Aufgabe der Fleischkochapparate ist, gleichzeitig aber auch ihre schwierigste, da die anderen im Fleisch vorkommenden pathogenen Bakterien ebenso wie Finnen und Trichinen leichter als die Tuberkelbacillen durch Erhitzen zu vernichten sind. Die Infection des Fleisches mit Tuberkelbacillen war der Regel nach eine künstlich vollzogene. Zwar stand zu den meisten Versuchen Fleisch tuberculös erkrankter Thiere zur Verfügung. Da aber die tuberkelbacillenhaltigen Krankheitsproducte (Perlknoten in Pleura und Peritoneum, erkrankte Lymphdrüsen) meist an der Oberfläche der Fleischstücke sich befinden, wo die Bacillen leicht abtödtbar sind, während die Anwesenheit von Tuberkelbacillen im Centrum der Stücke, in der Fleischmasse selbst, zweifelhaft ist, so wurde gewöhnlich eine künstliche Infection des Fleisches rorgenommen. Nur gelegentlich einmal wurde durch Zusammenrollen von Bauchmuskel- und Zwerchfellstücken, mit den tuberculösen Peritonealüberzügen nach innen $z \mathfrak{u}$, eine Roulade hergestellt und in Versuch genommen, bei der dann natürlich

${ }^{1}$ Vgl. Hertwig, Deutsche Vierteljahresschrift für öffentl. Gesundheitspflege. Bd. XXIV. Hft. 3. - Duncker, Zeitschrift für Fleisch-und Milchhygiene. 1891. Bd.II. S.21. - Maske, Ebenda. 1893. Bd.III. S. 115. - Rieck, Archiv für wissenschaftl. u. pralct. Thierheilkunde. Bd. XXI. Hft. 2 u. 3. - Lieb e, Zeitschrift für Fleisch- $u$. Milchhygiene. 1894. Bd. IV. S. 143. 
eine künstliche Infection der centralen Partieen unterbleiben konnte. Zur Infection der Fleischstücke mit Tuberculose dienten Reinculturen von Tuberkelbacillen, tuberculöse Sputa vom Menschen und tuberculöse Organstückchen von Mensch, Rind, Schwein und Meerschweinchen. Das Infectionsmaterial wurde gewöhnlich, um es nach der Kochung leicht wieder im Fleische auffinden zu können, in eine einfache Schicht Fliesspapier eingewickelt in die Mitte der Fleischstücke oder in besonders geschützte Partieen derselben, z. B. auf platten Knochen aufliegende Muskelpartieen gebracht. Behufs Einführung des Infectionsmateriales wurde mittels des zum Duncker'schen Läuteapparate gehörigen Fleischbohrers oder eines langen, schmalen Fleischermessers ein Schlitz bis in die gewünschte Tiefe des Fleischstückes gebohrt. Nach Einschieben des Infectionsmateriales wurde der Schlitz durch Zusammenbinden des Fleisches oder, was sich als gleich wirksam erwies, durch festes Zusammendrücken desselben wieder geschlossen. Nach erfolgter Kochung wurde das in die Fleischstücke eingebrachte Tuberculosematerial aus denselben herausgeschnitten, worauf Theile desselben von doppelter Grösse einer Erbse im Laboratorium Meerschweinchen in eine Tasche des Unterhautzellgewebes am Bauche eingenäht warden. Die so geimpften Thiere wurden unter beständiger Aufsicht gehalten, etwa 8 Wochen nach der Impfung, soweit sie nicht vorher eingegangen waren, dureh Genickschlag getödtet und sorgfältigst auf das Vorhandensein tuberculōser Veränderungen untersucht. Fanden sich bei einem Thiere Erscheinungen, welche Tuberculoseverdacht rechtfertigten, so wurden neben der mikroskopischen Untersuchung Cultur- und Infectionsversuche mit den verdächtigen Eruptionen oder Organtheilen unternommen, um Klarheit zu schaffen, ob wirklich Tuberculose oder eine ähnliche Erkrankung vorlag.

In jedem Versuche wurde auch wenigstens je ein Meerschweinchen mit ungekochten Theilen der zur Fleischinfection dienenden Materialien zur Controle subeutan inficirt. Nur wenn dies Controlthier tuberculös erkrankte, wurde der Versuch als. Experiment über die Beeinflussung von Tuberkelbacillen durch die Kochung verwerthet. So muss z. B. Versuch III bezüglich der Tuberculose ausser Betracht bleiben, weil das Controlthier nicht an Tuberculose erkrankte.

Ausserer Tuberkelbacillen kamen zur Infection von Fleischstücken zur Verwendung: An Seidenfäden angetrocknete Milzbrandsporen von 5 bis 6 Minuten Resistenz gegen strömenden Wasserdampf von $100^{\circ}$, Milzbrandbacillen, Staphylococcus pyogenes aureus und albus aus Abscessen rom Menschen und vom Rind, Bacillen des Schweineroth. laufs und der Schweineseuche, Bacterium coli commune, Bacillus bovis morbificans (Basenau) und ein dem Bacillus enteri- 
tidis gleichender Mikroorganismus, den ich aus einem durch Genuss gehackten rohen Rindfleisches veranlassten Falle von Fleischvergiftung gezüchtet habo.

Die Verwendung von Milzbrandsporen wurde nur deshalb beliebt, weil dieselben ein zweckmässiges Testobject bilden. Sind selbst Milzbrandsporen in einem Fleischstücke vernichtet, so ist der Kocherfolg als ein sehr guter zu bezeichnen. Andererseits beweist das Ueberleben der Milzbrandsporen aber nicht, dass der Kocheffect schlecht gewesen ist, denn, trotzdem die Milzbrandsporen überleben, können die anderen, praktisch als im Fleische abzutödtend in Frage kommenden Bakterien wohl zu Grunde gegangen sein.

Als Testobject nach der anderen Seite hin sollten die sporenfreien Milzbrandbacillen dienen. Die Kochung war unbedingt nicht genügend, wenn selbst diese so leicht abzutödtenden Bacillen dieselbe lebend überdauert hatten, - ein Ereigniss, das übrigens niemals beobachtet wurde.

Die übrigen Mikroorganismen sind solche, mit denen zur Kochung in den Apparaten bestimmtes Fleisch inficirt sein kann. Das Fleisch wurde mit innen in derselben Weise, wie sie oben bezüglich der Tuberkelbacillen besehrieben worden ist, beschickt. Die Mikrobien kamen entweder in Culturen zur Verwendung oder aber in Organstückchen bezw. Eiter von Versuchsthieren, deren Tod eine Infection mit Reinculturen der betreffenden Organismen veranlasst hatte. Selbstrerständlich wurde immer durch Mikroskop und Cultur die reichliche Gegenwart der Organismen in dem Infectionsmateriale des Fleisches vor der Kochung nachgewiesen. Culturmaterial und Eiter wurde auf Wattebäuschchen aufgestrichen und, in Fliesspapier gehüllt, eingebracht; Organstücke wurden, in Fliesspapier verpackt, in die Fleischstücke eingeführt. Nach der Kochung herausgenommen, wurden die bakterienhaltigen Wattebäuschchen oder Organstückchen in Nährbouillon ausgesät und mindestens 14 Tage lang bei $35^{\circ}$ bebrütet. Sobald in einem Röhrchen Wachsthum eintrat, wurde durch Plattenculturen und geeigneten Falles durch Thierversuche die Natur der entwickelten Mikrobien bestimmt.

Ausser den pathogenen Bakterien wurden in die Fleischstücke vielfach auch zum $\mathrm{Zwecke}$ vergleichender Untersuchungen Maximalthermometer und Läutewerke, die verschiedene Temperaturen (zwischen 70 und $100^{\circ}$ ) signalisirten, eingebracht, und zwar wurden sie in die Mitte der Fleischstücke oder die sonst anscheinend am schwersten zu erhitzenden Partieen derselben eingebohrt.

Die zu den Versuchen benutzten Fleischstücke stammten von Rind, Schwein und Hammel und darunter von alten und jungen, mageren und fetten Thieren. Sie wurden in Stücke von 700 bis 6000 grm Gewicht zer- 
legt und in die Korbeinsätze der Apparate, wie bei den Kochungen zum Verkaufe, derart eingepackt, dass die einzelnen Fleischstücke möglichst mit kleinen Zwischenräumen neben, nicht über einander lagen. Der Kochprocess wurde in der Weise ausgeführt, dass nach Beladung und Schliessung des Kessels zunächst bei der Anheizung desselben der in das Kesselinnere führende Luftentwickelungshahn so lange geöffnet blieb, bis aus ihm in scharfem gleichmässigen Strahle feucht und heiss sich anfühlender Dampf ausströmte. Alsdann wurde der Hahn geschlossen, durch Stellung des Dampfventils die Erhitzung des Kessels regulirt und der Druck in seinem. Innern auf die gewünschte Höhe gebracht. Die Kochdauer wurde von dem Momente der Lufthahnschliessung an gerechnet. Variirt wurde in den Versuchen ausser der Art des zu kochenden Fleisches und der zur Infection dienenden Bakterien die Grösse der Fleisch. stücke, die Länge des Kochprocesses und die Höhe des im Kesselinnern herrschenden Druckes.

Nach Ablauf der gewählten Kochdauer wurde der Heizdampf abgestellt, der Apparat sofort geöffnet und entladen.

Zahlreiche Fleischstücke wurden vor der Kochung und sofort nach derselben oder nach dem Erkalten am nächsten Morgen wieder gewogen, um ein Urtheil über die durch das Kochen verursachten Gewichtsverluste zu gewinnen. Die Wägung der erkalteten Stücke musste bisweilen, und zwar dann unterbleiben, wenn das Fleisch sofort der Abdeckerei ausgeliefert werden musste; aber auch aus dem Gewicht der noch warmen Stücke, das nach Maassgabe der Wägungen in den anderen Versuchen immer um wenige Procente höher als das der erkalteten ist, lässt sich ein Schluss auf den Totalgewichtsverlust ziehen, den das Fleisch vom rohen Zustande bis zum Verkaufe erlitten haben würde, der in Hamburg der Regel nach am Tage nach der Kochung, wenn das Fleisch völlig. erkaltet ist, stattfindet.

Alle za den Versuchen verwendeten Fleischstücke wurden der $A b-$ deckerei übergeben oder durch Feuer vernichtet. Die in dem Apparate nach Herausnahme des Fleisches zurückbleibende Brühe wurde nochmals 2 Stunden lang bei 1 Atmosphäre gekocht, ehe der Apparat wieder eine Kochung zu Verkaufszwecken ausführte.

Alle Einzelheiten der Versuche ergeben sich aus den beigefügten, in Tabellenform gebrachten Protocollen über dieselben, denen eine specielle, Erläuterung auf S. 405 vorausgeschickt ist. 
V.

Aus den Versuchstabellen ergiebt sich zunächst, in voller Bestätigung der oben ausgeführten Ueberlegungen, wie misslich es ist, von der in ein em Fleischstücke erreichten Temperaturhöhe einen Rückschluss auf die in den anderen erzielte Temperatur zu ziehen. Scheinbar leicht permeable Stücke hatten häufig niedrigere Temperaturen als andere, deren Durchdringung, wie man annehmen sollte, grössere Schwierigkeiten hätte bieten müssen. So erreicht z. B. in Versuch XII das 2000 grm schwere Stück 2 erst nach 1 Stunde 2 Minuten $70^{\circ}$, während das um die Hälfte schwerere Stück 1 schon nach 55 Minuten $70^{\circ}$ im Innern signalisirt. In Versuch XI hat das 1800 grm schwere Stück 3 nach 1 Stunde, das fast doppelt so schwere Stück 2 nur 5 Minuten später $70^{\circ}$ im Innern. Jeder Versuch zeigt die mangelnde Proportionalität zwischen Grösse der Fleischstücke und in ihnen erreichter Temperaturhöhe. Ich greife Versuch XXII als Beleg heraus: In Stück 1, Schweinerücken von $2900 \mathrm{grm}$, sind $99^{\circ}$ erreicht, Stück 2 und Stück 5, ebenfalls Schweinerücken, von 2750 und $2450 \mathrm{grm}$ Gewicht, zeigen dagegen nur $85^{\circ} \mathrm{im}$ Centrum. Im Schinkenstück 7 desselben Versuches von $4000 \mathrm{grm}$ Gewicht sind $94^{\circ}$ erstiegen, während in den nur 3350 bezw. $2925 \mathrm{grm}$ schweren Schinken 9 und 10 die Temperatur nur 80 und $87^{\circ}$ beträgt. - Interessante Resultate liefert Stück 2 in Versuch XIV. In die Mitte dieses Stückes waren von zwei Seiten her Temperaturmessinstrumente eingeführt worden, und zwar ein Maximalthermometer und ein $80^{\circ}$ meldendes Contactthermometer. Das Maximalthermometer zeigte nach Beendigung der Kochung nur $76^{\circ}$, während das ebenfalls in der Mitte des Stückes, wenige Centimeter von dem Maximalthermometer entfernt gelegene Contactthermometer bereits 23 Minuten vor Schluss $80^{\circ}$ gemeldet hatte. In ganz nahe benachbarte Partieen im Centrum eines Fleischstückes kann daher die Temperatur sehr verschieden schnell eindringen, folglich auch darin recht verschiedene Höhe erreichen. In gleichem Sinne spricht z. B. Versuch XX Stück 13, in dem das in die Mitte eingeführte Thermometer $87^{\circ}$ zeigt, während das Fleisch an einer anderen Stelle des Centrums noch blutig ist, also dort noch nicht einige Minuten lang $70^{\circ}$ gehabt haben kann. ${ }^{1}$ Jeder Versuch liefert Beweise dafür, dass Fleischstücke, in denen die Temperatur weit unter $100^{\circ}$ bleibt, völlig gar und zart gekocht sein können.

$170^{\circ}$ gilt als die Temperaturgrenze, bei der sich der Blatfarbstoff von Roth in Braun verwandelt. Mit Blat und blutigen Fleischstücken angestellte Erhitzungsversuche zeigten mir, dass $60^{\circ}$ selbst bei Stunden langer Einwirkung den Blutfarbstoff nicht verändert, $70^{\circ}$ ettwa in $3 \mathrm{Min} .8^{\circ}$ momentan ihn in Braun umwandelt. 
Aus alledem folgt, wie richtig die Erwägung war, nicht auf die Höhe der beobachteten Temperaturen allein sein Augenmerk zu richten und eine Vorschrift für das Kochverfahren zu basiren, sondern den umständlicheren, aber zuverlässiger zum Ziele führenden Weg der Beobachtung zu wählen, bej. welcher Betriebsart die pathogenen Mikrobien des Fleisches, unter möglichster Schonung der Beschaffenheit des Fleisches selbst, sicher abgetödtet werden können.

In erster Linie kommt dabei der Erreger der Tuberculose in Betracht. In den 23, mit Einbringung von Bakterienmaterial ausgeführten Kochversuchen wurden 180 Fleischstücke mit tuberculösen Laterialien inficirt und damit 181 Meerschweinchen geimpft. (Mit Material von einem Stücke wurden zwei Meerschweinchen, sonst immer je eins inficirt.) Von diesen 181 Meerschweinchen sind 170 für die Beurtheilung des Einflusses der Kochung auf die Tuberkelbacillen zu verwerthen, die übrigen 11 nicht, und zwar 2 deshalb nicht, weil das zugehörige Controlthier, mit ungekochtem Tuberculosenmateriale geimpft, nicht an Tuberculose erkrankte, die anderen 9 aber darum nicht, weil sie an intercurrenten Krankheiten (Pseudotuberculose, Sepsis, unbekannte Erkrankungen) so bald nach der Infection verstarben, dass die etwa ihnen eingeimpften lebenden Tuberkelbacillen noch keine Tuberculoseerscheinungen im Körper der Thiere hatten hervorbringen können. Eins dieser 9 Thiere (Versuch XX, Stück 11) starb 14 Tage nach der Impfung an Tubereulose. Dieselbe konnte aber nicht als' Effect der Impfung angesehen werden, denn erstlich waren die Impfstelle und die ihr benachbarten Drüsen ganz reactionslos, zweitens aber die tuberculösen Processe in den inneren Organen so vorgeschritten, dass sie sich unmöglich innerhalb 14 Tage entwickelt haben konnten. Es handelte sich um ein aus einem Krankenhause kurz vor der Impfung angekauftes Thier, das schon zur Zeit der Impfung, wenn auch nicht wahrnehmbar, tuberculös gewesen sein muss.

Die 170 Meerschweinchen, welche das Material für die Beurtheilung der Kochwirkung auf die Tuberkelerreger bilden, waren mit tubereulösem Materiale aus 169 Fleischstücken geimpft worden. Diese 169 Fleischstücke stammten von Rind, Schwein und Schaf. Ihre Grösse schwankte etwa zwischen 700 und 6000 grm, die Zeitdauer ihrer Kochung zwischen 1 Stunde und $2^{1} / 2$ Stunden. Als Resultat ergab sich Folgendes: In 168 Stücken war, wie das Freibleiben der Meerschweinchen von Tuberculose ergab, die Infectiosität des tuberculösen Materiales oder, was dasselbe sagen will, das Leben der Tuberkelbacillen vernichtet worden. Nur das Tuberculosematerial aus einem Stücke erwies sich noch als infectiös. Es handelte sich hier um einen Schinken von 4600 grm (Versuch XXI, Stück 4), der 11/2 Stunden bei $1 / 2$ Atmosphäre Ueberdruck gekocht worden, dabei aber in der Mitte 
noch roh geblieben war und dort nur eine Temperatur von $59^{\circ}$ erreicht hatte. 5 andere, durchweg noch etwas schwere Stücke desselben Versuches batten dagegen bei gleicher Behandlung keine lebenden Tuberkelbacillen nach der Kochung mehr beherbergt und auch höhere Temperaturen im Innern erreicht.

Abgesehen von diesem einen Falle waren die Tuberkelbacillen immer abgetödtet worden. Schon $1 \frac{1}{2}$ stündiges Kochen hatte sie bei $1 / 2$ Atmosphäre Ueberdruck selbst in Stücken Rind- und Schweinefleisch bis gegen 6 kg Schwere immer vernichtet (vgl. Versuche $\mathrm{XX}$ und $\mathrm{XXI}$ ). In einem Versuche (V) war nur 1 Stunde lang bei $1 / 2$ Atmosphäre Ueberdruck gekocht worden. Auch hier waren die Tuberkelbacillen im Innern der beiden in Versuch genommenen, 4950 bezw. 1850 grm schweren Fleischstücke abgetödtet worden, trotzdem die Temperatur in dem grösseren Fleischstücke noch unter $70^{\circ}$ geblieben war und selbst in der Mitte des kleineren Stückes $72.5^{\circ}$ nicht überschritten hatte. In Agar-Agar geschützt eingebettete Eiterstaphylokokken hatten dagegen in diesem Versuche noch überlebt!

Kann man nach dem Ausfalle der Experimente annehmen, dass der Regel nach Tuberkelbacillen selbst im Innern von Fleischstücken bis zu 6000 grm Gewicht bei $11 / 2$ stündiger Erhitzung schon unter $1 / 2$ Atmosphäre Ueberdruck zu Grunde gehen, so lässt doch der eine Misserfolg in Versuch XXI diese Kochdauer nicht für alle Fälle als genügend ansehen, vielmehr eine Kochung über 2 Stunden hin, bei der niemals ein Ueberleben der Tuberkelbacillen beobachtet wurde, als erforderlich zur Erzielung sicherer Abtödtung erscheinen.

Bestimmte Beobachtungen legen es aber auch nahe, das Gewicht der Fleischstücke nicht gar zu hoch zu wählen. Zwar sind bei 2 stündiger Kochdauer auch in den grössten verwendeten Fleischstücken die Tuberkelbacillen überall abgetödtet worden. Indessen zeigt eine Durchsicht der Tabellen, dass in manchen der grössten Fleischstücke bei 2 stündiger Kochung noch Stellen vorhanden waren, die röthliche Färbung besassen, also kaum oder nur für kurze Zeit die Temperatur von $70^{\circ}$ erreicht hatten, die man grösserer Sicherheit halber doch wohl verlangen muss, um der Vernichtung der Tuberkelbacillen sicher zu sein. Andere Stellen der grössten Stüeke waren durch die Kochung noch nicht ganz zart gekocht worden, genügten mithin nicht den an eine gute Kochung zu stellenden Anforderungen. Um der Abtödtung der Tuberkelbacillen ganz sicher sein zu können, wird man das Grössemaximum der Fleischstücke zweckmässig auf $3000 \mathrm{grm}$ normiren. Stücke bis zu dieser Grösse kochen, wie die Tabellen zeigen, regelmässig bis in's Innerste rollkommen gar. Dass einzelne, übrigens schon im rohen Zustande leicht kenntliche Stücke, 
Kochapparate FŨR BEDINGT GESUNDHEITSSCHÄDLICHES FLEISCH. 399

selbst bei dieser Maximalgrösse dem Eindringen der Hitze noch erheblichen Widerstand entgegen setzen, daher noch weiter zu zertheilen sind, wird unten noch des Näheren erörtert werden.

Kommt man also zu dem Schlusse, dass zur Abtōdtung der Tuberkelbacillen die Stücke zweckmässig nicht grösser als $3000^{\mathrm{grm}}$ gesehlagen und 2 Stunden lang gekocht werden, so bleibt des Weiteren noch zu prüfen, ob auch die anderen pathogenen Fleischbakterien bei dieser Kochmethode sicher abgetödtet werden und ferner, ob auch das Fleisch dabei eine gute Beschaffenheit behält, bezw. erhält.

Die neben den Tuberkelbacillen als Eindringlinge im Fleische in Betracht kommenden Bakterien - und ebenso die oben erwähnten thierischen Parasiten, mit denen aber nicht experimentirt wurde - sind, wie schon besprochen wurde, weit weniger resistent gegen Einwirkung erhöhter Temperaturen als die Tuberkelbacillen. Die in den Versuchen verwendeten Bakterien wurden noch speciell auf ihre Widerstandsfähigkeit gegen Erhitzen in Aussaaten, Culturen und Organstücken von inficirten Thieren im Laboratoriumsversuche geprüft. $\mathrm{Zu}$ dem Behufe wurden Bouillonröhrchen, in welchen Aussaaten von Culturen oder die Bakterien enthaltende Thierorganstücke eingebracht waren, in genau eingestelltem Wasserbade, unter steter Controle der Temperatur in den Röhrchen selbst, auf Temperaturen von 60 und $70^{\circ}$ verschieden lange Zeit hindurch erwärmt und dann 8 Tage lang bei $35^{\circ}$ bebrütet. Ferner wurden entwickelte junge Bouillonculturen ebenso erhitzt, darauf 3 Tage lang bebrütet und alsdann cubikcentimeterweise in sterile Bouillon übertragen und mit dieser auf's Neue bebrütet; dieses Verfahren wurde gewählt, um auch einzelne, etwa überlebende Bakterienkeime noch sicher nachweisen zu können. ${ }^{1}$ Es ergab sich, dass alle zu den Versuchen gebrauchten Bakterienarten, mit Ausnahme natürlich der Milzbrandsporen, durch Erhitzen auf $60^{\circ}$ in wenigen Minuten bereits getödtet wurden. Nur die aus einer Fleischvergiftung gezüchteten Bacillen überlebten gelegentlich sogar Temperaturen von $70^{\circ}$ ein Paar Minuten.

Entsprechend dem Ausfalle dieser Laboratoriumsversuche waren die pathogenen Bakterien (ausser den Milzbrandsporen) auch in den Kochversuchen im Innern der dicksten Fleischstücke stets zu Grunde gegangen. Nur in Versuch V hatten in Agar-Agar eingebettete Staphylokokken, wie schon erwähnt, 1 stündiges Erhitzen der Fleischstücke überdauert. Ferner waren in Versuch XXI, Stück $2(4700 \mathrm{grm})$ nach $1 \frac{1}{2}$ stündigem Kochen bei $1 / 2$ Atmosphäre Ueberdruck Schweinerothlaufbacillen noch am Leben ge-

1 Vgl. Toptschieff, Centralblatt für Bakteriologie. Abth. I. Bd. XXXIII. S. 733. 
blieben, trotzdem ein Thermometer im Fleischinnern $79^{\circ}$ anzeigte. Wie es gekommen ist, dass die Rothlaufbacillen hier überdanert haben, ist nicht ganz klar. Es kann sich vielleicht um zufällig hervorragend resistente Bacillen gehandelt haben, wie sie auch $P_{\text {etri }}{ }^{1}$ ganz unvermittelt zwischen wenig widerstandsfähigen in seinen Rothlaufuntersuchungen beobachtet hat. Vielleicht auch mag ihre Lage im Fleische sie besonders geschützt haben. Das Stück Fleisch war ein Schulterstück, an dem das Schulterblatt eine Art von Schutzdecke gegen das Eindringen der Hitze bildete. Wie noch zu besprechen sein wird, sind solche flachen Knochen, wie Schulterblatt und Beckenschaufel, ganz besonders geeignet, das Eindringen der Hitze in's Fleisch zu verzögern. Auch in dem fraglichen Stücke scheinen nach der Beschaffenheit seiner Schnittfäche nicht überall Temperaturen von $70^{\circ}$ geherrseht zu haben. Kurz, es kann sein, dass die an sich vielleicht in diesem Falle sehr resistenten Rothlaufbacillen entfernt von der Lagestelle des $79^{\circ}$ zeigenden Thermometers im Schutze des platten Schulterknochens ein Asyl gegen die Hitzewirkung gefunden haben. Es folgt daraus nur, dass man auf die Zurichtung der platte Knochen enthaltenden Stücke specielle Sorgfalt verwenden muss, um das Eindringen der Hitze in alle ihre Theile zuverlässig zu erreichen.

Zum Abtödten der für das Fleisch in Frage kommenden pathogenen Bakterien, mit welchen in den Versuchen experimentirt worden ist, genügt nach den Ergebnissen der Untersuchungen 2 Stunden lange Einwirkung eines unter $1 / 2$ Atmosphäre Ueberdruck stehenden Dampfes auf Fleischstücke bis zu $3000 \mathrm{grm}$ Gewicht, also die Kochmethode, welche für die Vernichtung der Tuberkelbacillen als ausreichend befunden wurde, augenscheinlich vollkommen. Wie besonders Versuch XVIII sehr deutlich darthut, wie aber auch aus anderen Versuchen resultirt, können sogar Milzbrandsporen in den Fleischstücken bei dieser Art der Kochung (sogar in $4000 \mathrm{grm}$ schweren Stücken gelegentlich) vernichtet werden. Allerdings ist das nicht immer und überall der Fall (siehe z. B. Versuch XIII, wo hier und da Milzbrandsporen überleben), soll ja aber auch nicht in praxi durch die Kochung erreicht werden, da milzbrandiges Fleisch kein Kochobject wird, bekanntermassen auch nur Bacillen, keine Sporen enthält. ${ }^{2}$

1 Petri, Arbeiten aus dem Kaiserl. Gesundheitsamte. Bd. VI. S. 271.

2 Die verwendeten Milzbrandsporen überlebten 5 bis 6 Min. Dampf von $100^{\circ}$, waren also gut resistent. Bei Erhitzung in Bouillon auf $90^{\circ}$ lebten sie noch nach einer halben Stunde, nicht mehr nach einer Stunde. Je höher die Temperatur von $90^{\circ} \mathrm{zu} 100^{\circ}$ heranrückte, desto schneller gingen sie zu Grunde. In den Versuchen von $\mathrm{Koch}$, Löffler and Gaffky (Mittheilungen aus dem Kaiserl. Gesuñdheitsamte, Bd. I, S. 322) worden Milzbrandsporen durch Dampf von $9^{\circ}$ in 10 Min. vernichtet. 
Nach den Resultaten der bakteriologischen Versuche würde demnach die angegebene Art der Kochung zu empfehlen sein. Es ist nun aber weiter die Frage, ob auch das Fleisch dabei in guter Weise gekocht, d. h. gar und zart wird, saftig bleibt, nicht so stark zerfällt, dass die Knochen sich herauslösen und nicht einen zu hohen Procentsatz an Gewicht verliert.

Bei Beurtheilung dieser Verhältnisse ist in erster Linie zu berücksichtigen, dass Fleisch und Fleisch etwas sehr Verschiedenes sein kann. Das Fleisch vollsaftiger junger Rinder kocht viel leichter gar als das alter abgemagerter, das Fleisch magerer Schweine leichter als das sehr fetter, das Fleisch von Schweinen meist etwas leichter als das von Rindern. Im Einzelnen zu entscheiden, wie lange gekocht werden muss, um alle Fleischstücke gar zu erhalten, muss in das Ermessen der die Kochung leitenden Person gestellt, von ihrer Erfahrung abhängig gemacht werden. Die auf die bakteriologischen Resultate basirte Vorschrift stellt nur das unumgänglich einzuhaltende Mindestmaass der Kochdauer und das nicht zu überschreitende Höchstmaass des Fleischstückgewichtes fest. Bei schwer zu durchkochendem Fleische kann der Leiter der Kochung selbstrerständlich jenes Mindestmaass überschreiten und unter diesem Höchstmaasse zurückbleiben, um sichere Garkochung zu erzielen, d. h. länger kochen und die Fleischstücke kleiner wählen. Dass die rorgeschlagene Kochmethode selbst schwer zu durchkochendes Fleisch in gut garem Zustande liefert und selbst leicht zu durchkochendes nicht zu sehr angreift, hat sich in praxi gezeigt.

$\mathrm{Zu}$ bedenken sind noch folgende Punkte:

a) Fleischstücke, an oder in denen sich platte Knochen (Schulterblatt, Becken) befinden, sind oft schwer zu durchkochen, daher in kleinere als $3000 \mathrm{grm}$ schwere Portionen zu zerlegen. Als Belege für die schwere Durchkochung vergleiche man z. B. Versuch XIV, Stūck 12 und Versuch XIX, Stück 11 (niedrige Temperatur!) mit den anderen Stücken in den gleichen Versuchen.

b) Sehr fettes Fleisch, besonders Schweinefleisch, setzt, zumal wenn an ihm noch die Schwarte erhalten geblieben ist, dem Eindringen der Hitze sehr erheblichen Widerstand entgegen, ist daher ebenfalls in kleinere Portionen als 3000 grm zu zerlegen (s. hierzu Versuch XIV).

c) Schiere Fleischstücke sind, wie auch Duncker ${ }^{1}$ beobachtet hat,

1 Duncker, Zeitschr. für Fleisch-und Milchhygiene. 1891. Bd. II. S. 21. Petri (a. a. O., S. 273) fand, dass die Hitze anscheinend vorzüglich den Knochen entlang eindringt. Dies stimmt $z \mathfrak{u}$ den Experimentalergebnissen von Landois ( Lehrbuch der Physiologie des Menschen, 5. Aufl., 1887, S. 404), dass von allen Körpergeweben die Knochen die besten Wärmeleiter sind. Dass Stücke mit platten Knochen, wie unter a) bemerkt, schwer durchkochen, erklärt sich vielleicht ans ihrem hohen Gehalt an Sehnen and straffem Bindegewebe.

Zeitschr. f. Hygiene. XXX. 
im Allgemeinen schwerer für Hitze permeabel als knochenhaltige Stücke. (So z. B. Versuch XVIII, Stück 1, 2 und 5 und die zugehörigen Temperaturen.) Daher schneide man schiere Fleischstücke statt zu 3000 nur zu 2000 grna.

Unter Berücksichtigung dieser Punkte wird es dem erfahrenen Leiter der Kochung unter Zugrundelegung der angegebenen Vorschrift immer gelingen, das Fleisch gut gar zu kochen, ohne dass ein zu starkes Auskochen des Fleisches oder gar ein Zerfallen und Unansehnlichwerden desselben zu befürchten ist. $\mathrm{Ob}$ das Fleisch in den Körben der oberen, der beiden mittleren oder der unteren Reihe untergebracht war, machte bezüglich der Schnelligkeit der Durchkochung keinen Unterschied.

Einer kurzen Erörterung bedarf noch die Frage, ob es zweckmässig ist, mit Ueberdruck von $1 / 2$ Atmosphäre oder mit Ueberdruck von 1 Atmosphäre zu arbeiten. Man könnte erwarten, dass man bis 1 Atmosphäre Ueberdruck mittels der einwirkenden höheren Temperatur schnellere und gründlichere Durchkochung der Fleischstücke erreicht. Nach dieser Richtung haben die Versuche gezeigt, dass das Eindringen der Hitze in die Fleischstücke anscheinend bei 1 Atmosphäre Ueberdruck Anfangs nicht schneller als bei $1 / 2$ Atmosphäre Ueberdruck erfolgt (vgl. Versuche VIII, IX, XI, XII, XIIII, XIV, XV, XVI, XXIII). Erst wenn in den Stücken eine Temperatur von 80 bis $90^{\circ}$ erreicht ist, scheint die bei 1 Atmosphäre Ueberdruck herrschende höhere Temperatur sich stärker wirksam zu erweisen. So sind die schliesslich bei 1 Atmosphäre Ueberdruck im Fleischinnern erzielten Temperaturen höher als die bei $1 / 2$ Atmosphäre Ueberdruck erreichten. Aber die Kochresultate sind, wie die Parallelversuche XVII und XVIII, XIX und XX, XXII und XXIII darthun, günstigere bei Ver. wendung von $1 / 2$ Atmosphäre als von 1 Atmosphäre. In diesen Versuchen verlor das Fleisch bei $1 / 2$ Atmosphäre Ueberdruck 39 Procent (Rindfleisch), bezw. 38 Proc. (Rindfleisch), bezw. 18.7 Proc. (Schweinefleisch) an Gewicht, während von den gleichen Thieren stammendes Fleisch bei 1 Atmosphäre Ueberdruck einbüsste: '46 Procent (Rindfleisch), bezw. 40 Procent (Rindfleisch), bezw. 27.4 Procent (Schweinefleisch). Bei $1 / 2$ Atmosphäre Ueberdruck ist also der Gewichtsverlust geringer ${ }^{1}$, und abgesehen von der dadurch gegebenen höheren Rentabilität das Fleisch auch saftiger, folglich wohlschmeckender. Bei den Versuchen mit 1 Atmosphäre Ueberdruck wurden einzelne Stüeke bereits stark zerkocht, also hinsichtlich der Ver-

1 Eine Ausnahme machen Versuch XVI bis XVII, in denen 1 Atm. Ueberdruck 46 Procent Gewichtsverlust, $1 \% \mathrm{Atm}$. dagegen 47 Procent veranlasst hat. In diesen Versuchen ist die Kochdauer ( $2{ }^{1} / 2$ Stunden) im Verhältniss zu der Beschaffenheit des Fleisches überhaupt eine zn lange gewesen. Auch hier sind aber bei $1 \mathrm{Atm}$. Ueberdrack eine grössere Zahl von Stücken als bei $1 / 2$ Atm. stark zerkocht gewesen. 
werthbarkeit minderwerthig gefunden. Es besteht demnach kein Grund, zu 1 Atmosphäre Ueberdruck zu greifen, zumal auch die Abtödtung der pathogenen Bakterien bei Anwendung von $1 / 2$ Atmosphäre Ueberdruck und Beobachtung der bespruchenen Kochdauer und Fleischstückgrösse gewährleistet wird. In einigen Versuchen wurde in Anlehnung an ein in der Hamburger Kochanstalt für Verkaufskochungen befolgtes Verfahren zum Schluss des Kochprocesses der Druck im Kessel für $1 / 4$ Stande von $1 / 2$ auf $3 /{ }_{4}$ Atmosphäre gesteigert. Besondere Vortheile dieses Verfahrens konnten nicht wahrgenommen werden; indessen ist es als nicht irrationell anzusehen, wenn man bei schwer permeablem Fleische gegen Ende der Kochung den Druck auf $3 / 4$ Atmosphäre steigert, um damit vielleicht die Durchkochung der Fleischstücke zu fördern. Uuter $1 / 2$ Atmosphäre herabzugehen, dürfte sich kaum empfehlen, da der Jeberdruck von $1 / 2$ Atmosphäre die Beschaffenheit des gekochten Fleisches in keinerlei Hinsicht wahrnehmbar ad pejus beeinflusst, jedenfalls aber die Durehkochung des Fleisches befördert.

Die Gewichtsverluste des Fleisches halten sich bei der vorgeschlagenen Kochmethode in mässigen Grenzen. Um ein genaues Urtheil über die Höhe der Verluste zu gewinnen, genügen die relativ kleinen, in den Versuchen verwendeten Fleischmengen nicht. Jedenfalls scheint die Höhe der Verluste nicht grösser als bei der Kochung nach anderen Vorschriften und in anderen Apparaten zu sein, ${ }^{1}$ sondern sich auf etwa 20 bis 30 Proc. für Schweinefleisch, 30 bis einige 40 Procent für Rindfleisch zu beziffern. Dass viel niedrigere Verluste überhaupt nicht zu erzielen sein werden, macht Versuch $V$ wahrseheinlich. Schon bei 1stündiger Kochung haben die dort gekochten Rindfleischstücke einen Verlust von 25 Procent ihres Gewichtes aufzuweisen. Da es überhaupt undenkbar ist, kürzere Zeit als 1 Stunde zu kochen, so wird man wenigstens für Rindfleisch einen VerIust von mindestens 25 Procent immer mit in Kauf nehmen müssen. Rubner ${ }^{2}$ giebt an, dass bei $100^{\circ}$ gekochtes Fleisch 40 bis 43 Procent seines Gewichtes verliert. Nothwang ${ }^{3}$ fand nach 1 stündigem Kochen 31.1 Procent, nach 2stündigem 45.4 Procent Gewichtsverlust. Aehnliche Werthe haben Ferrati's ${ }^{4}$ Versuche geliefert.

Das durch die Versuche als zweckmässig, sowohl was die sichere Abtödtung der pathogenen Bakterien im Fleische, als auch was ausreichende,

${ }^{1}$ Siehe Hertwig, Noack, Rieck, Liebe, a. a. O., Hartenstein, Zeitschr. für Fleisch- und Milchhygiene, 1895, Bd. V, S. 61 und Hengst, ref. Ebenda, 1898, Bd. IX, Nr. 1, S. 12.

${ }^{2}$ Rubner, Lehrbuch der Hygiene. 5. Aufl. S. 500.

${ }^{3}$ Nothwang, Archiv fuir Hygiene. Bd. XVIII. S. 80.

4 Ferrati, Ebenda. Bd. XIX. S. 317. 
aber nicht zu starke Durchkochung des Fleisches betrifft, befundene Kochverfahren lässt sich kurz in folgenden Vorschriften zusammenfassen:

1. Die zu kochenden Fleischstücke sollen an Gewicht $3000^{\mathrm{grm}}$ nicht überschreiten. Fleischstücke, welche platte Knochen enthalten, schiere Fleischstücke und sehr fette Schweinefleischstücke, diese namentlich, wenn von ihnen die Schwarte nicht entfernt ist, sollen nur die Hälfte oder zwei Drittel dieses Gewichtes besitzen. Die Fleischstücke müssen locker neben einander gepackt, dürfen nicht über einander geschichtet sein.

2. Der am Kochapparate angebrachte Lufthahn soll so lange offen bleiben, bis siedend heisser feuchter Dampf in gleichmãssigem Strahle aus ihm ausströmt. Nach Schluss des Lufthahnes soll die Kochung für Rind-, wie für Schweine- und Hammelfleisch wenigstens 2 Stunden dauern.

3. Der Druck im Kessel soll dabei $1 / 2$ Atmosphäre Ueberdrack betragen.

Wie für den Hartmann'schen Kochapparat, wird die Methode ohne Zweifel auch für alle ähnlich construirten Kochapparate, z. B. die von Rietschel und Henneberg und von Lümkemann-Dortmund gebauten Fleischkochapparate verwendbar sein.

Es ist selbstverständlich nichts dagegen einzuwenden, wenn es dem die Kochung Leitenden im Interesse guter Garkochung schwer zu kochenden Fleisches nöthig erscheinen sollte, die Fleischstücke kleiner als $3000 \mathrm{grm}$ zu wählen, und die Dauer der Kochung auf mehr als 2 Stunden zu verlängern oder auch den Ueberdruck im Apparate, namentlich gegẹn Ende der Kochung auf $3 / 4$ Atmosphäre zu erhöhen.

Als Regel sollte es gelten, nach beendeter Kochung einige der grōssten Fleischstücke zu durchschneiden. Sollten sie wider Erwarten nicht völlig gar gekocht sein, so sind auch alle ähnlich grossen Stücke zu durchschneiden und nochmals $1 / 4$ bis $1 / 2$ Stunde lang zu kochen. 